\title{
Dscam and Sidekick proteins direct lamina-specific synaptic connections in vertebrate retina
}

\author{
Masahito Yamagata ${ }^{1} \&$ Joshua R. Sanes ${ }^{1}$
}

Synaptic circuits in the retina transform visual input gathered by photoreceptors into messages that retinal ganglion cells (RGCs) send to the brain. Processes of retinal interneurons (amacrine and bipolar cells) form synapses on dendrites of RGCs in the inner plexiform layer (IPL). The IPL is divided into at least 10 parallel sublaminae; subsets of interneurons and RGCs arborize and form synapses in just one or a few of them ${ }^{1-3}$. These lamina-specific circuits determine the visual features to which RGC subtypes respond $^{3-5}$. Here we show that four closely related immunoglobulin superfamily (IgSF) adhesion molecules-Dscam (Down's syndrome cell adhesion molecule), DscamL (refs 6-9), Sidekick-1 and Sidekick-2 (ref. 10) - are expressed in chick by non-overlapping subsets of interneurons and RGCs that form synapses in distinct IPL sublaminae. Moreover, each protein is concentrated within the appropriate sublaminae and each mediates homophilic adhesion. Loss- and gain-of-function studies in vivo indicate that these IgSF members participate in determining the IPL sublaminae in which synaptic partners arborize and connect. Thus, vertebrate Dscams, like Drosophila Dscams ${ }^{11-19}$, play roles in neural connectivity. Together, our results on Dscams and Sidekicks suggest the existence of an IgSF code for laminar specificity in retina and, by implication, in other parts of the central nervous system.

We previously showed that Sidekick-1 and Sidekick-2, large transmembrane adhesion molecules of the IgSF, are expressed by complementary subsets of amacrine, bipolar and ganglion cells in chick retina ${ }^{10}$. Double-label in situ hybridization revealed that only 10-15\% of RGCs were positive for each Sidekick (Supplementary Table 1). We speculated that Sidekick-negative retinal neurons might express genes encoding related molecules. Homology searches of genomic databases revealed that their closest relatives are the Dscams ${ }^{6-9}$, which, like Sidekicks, contain multiple immunoglobulin and fibronectin type III domains, plus a carboxy terminus predicted to bind PDZ domains (Fig. 1a and Supplementary Fig. 1). Moreover, vertebrate Dscams are related to Drosophila Dscams, which have been implicated in neural specificity ${ }^{11-19}$, although they lack the complex pattern of alternative splicing found in Drosophila Dscam1. We therefore cloned chick Dscam and DscamL, the orthologues of mammalian Dscam and Dscam-like-1, respectively, and assessed their expression in retina. Dscam and DscamL, like Sidekicks, were expressed by non-overlapping subsets of retinal neurons between embryonic day (E) 12 and hatching (E21) (Fig. 1b and Supplementary Fig. 2). None of the Dscam-positive neurons expressed either Sidekick (Fig. 1c and Supplementary Fig. 3). Thus, Dscams and Sidekicks mark four distinct sets of presynaptic (amacrine and bipolar) and postsynaptic cells (RGCs) that arborize in the IPL.

To localize Dscam and Sidekick proteins, we generated antibodies specific for each (Supplementary Fig. 4) and used them to immunostain retinal sections. We follow the convention ${ }^{1-3}$ of dividing the IPL into 5 parallel slabs of equal width, S1-S5; each, however, may contain 2 or more sublaminae defined physiologically ${ }^{4,5}$. Each IgSF protein was concentrated in a distinct set of IPL sublaminae: Dscam in S5, DscamL in S1, S2 and S4, Sidekick-1 predominantly in the inner half of S4 with low expression in the inner half of S2, and Sidekick-2 predominantly in the inner half of S2 with low expression in the inner half of S4 (Fig. 1d). Confocal imaging showed that Dscams were frequently associated with vesicle-rich synaptic sites in the IPL (not shown), as shown previously for Sidekicks ${ }^{10}$. In situ hybridization of retinae in which individual cells had been filled with GFP (green fluorescent protein) by transposon-mediated gene transfer (see below) showed that neurites of cells expressing each Dscam or Sidekick gene arborize in IPL sublaminae containing the corresponding Dscam or Sidekick protein (Supplementary Fig. 5, ref. 10 and data not shown; summarized in Fig. 1e). Thus, Dscams and Sidekicks mark four distinct synaptic pathways in the retina.

Dscams, like Sidekicks, might not only mark lamina-specified neuronal subsets but also play roles in establishing or maintaining the sublaminar specificity of their synapses. We predicted that if this were the case, (1) each Dscam and Sidekick would mediate homophilic but not heterophilic adhesion; (2) depletion of Dscam or Sidekick would selectively perturb arbors of neurons in particular IPL sublaminae; and (3) ectopic expression of a Dscam or Sidekick would reroute arbors of the expressing cell to normally Dscam- or Sidekick-positive IPL sublaminae. We tested these ideas in turn.

To assess adhesion, we transfected heterologous cells with complementary DNAs encoding Dscams or Sidekicks, then allowed them to aggregate. Cells expressing any one of these four IgSF members formed aggregates but untransfected cells did not (Fig. 2a and data not shown). Moreover, Dscam and Sidekick proteins became concentrated at sites of contact between transfected cells (Fig. $2 \mathrm{~b}$ and data not shown). Thus, as shown previously $y^{7,8,10}$, Dscams and Sidekicks mediate homophilic adhesion. To assess heterophilic adhesion, we labelled transfected populations separately with red and green fluorescent dyes. When both populations expressed the same adhesion molecule, red and green cells co-aggregated to form mixed clusters, but when the two populations expressed different adhesion molecules, mixed aggregates were rare (Fig. 2a, c). Thus Dscams and Sidekicks do not detectably interact with each other under the conditions of our assay.

To decrease Dscam and Sidekick levels in retinal neurons, we identified interfering RNAs ${ }^{20,21}$ that targeted Dscam, Sidekick-1 and Sidekick-2 effectively (Supplementary Fig. 6) and incorporated them into retroviral vectors ${ }^{22}$ that also expressed GFP (Fig. 3a and b). Infection of retina with these vectors selectively decreased expression of the corresponding protein (Supplementary Fig. 7a). To assess effects of depleting Dscam or Sidekicks, we needed independent markers of the neurons that normally express them, so we could 

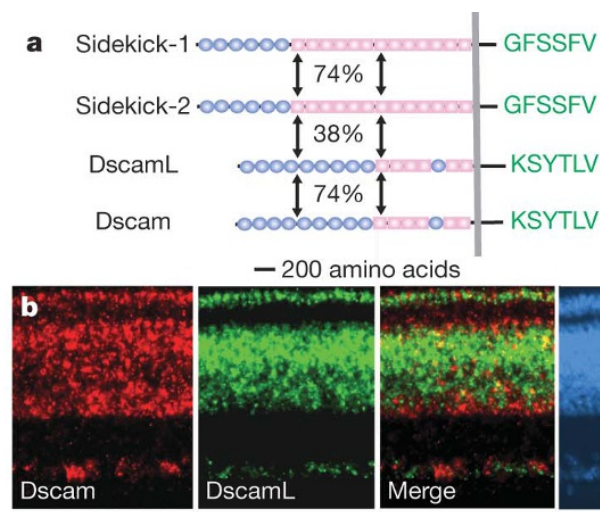

-200 amino acids
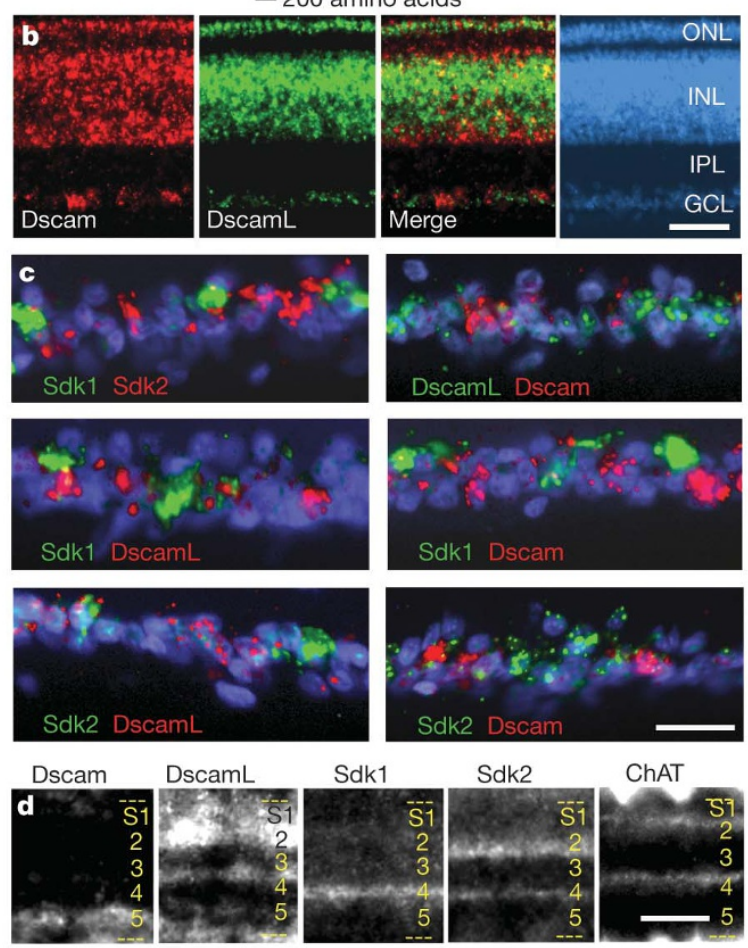

Sdk2

ChAT
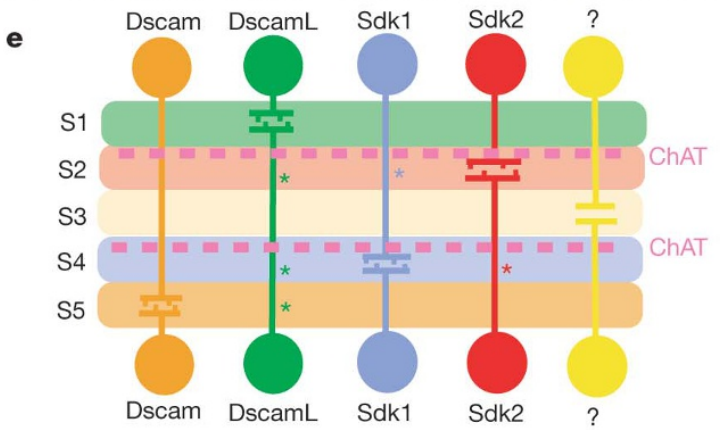

Figure 1 | Expression and distribution of Dscams and Sidekicks in retina. a, Structure of Dscam, DscamL and Sidekick (Sdk)-1 and -2. Blue, immunoglobulin domains; pink, fibronectin type III domains; grey, membrane; green, carboxy-terminal sequences predicted ${ }^{6-10}$ and shown (M.Y., unpublished data) to bind PDZ domains. Numbers show percentage amino acid similarity between indicated pairs of proteins, based on the Smith-Waterman algorithm applied to the entire sequence (see Methods). b, c, Double label in situ hybridization to sections of E15 retina showing that Dscam, DscamL, Sidekick-1 and Sidekick-2 are expressed by nonoverlapping subsets of neurons. Whole retina is shown in $\mathbf{b}$, ganglion cell layer only in c. Nuclear stain, DAPI, shown in blue. (See also Supplementary Fig. 3.) d, Immunostaining of retina with antibodies specific for Dscams, Sidekicks and choline acetyltransferase (ChAT). Each is concentrated in distinct sublaminae of the IPL. e, Summary of results from b-d and Supplementary Figs 2, 3 and 5, showing that each Dscam and Sidekick is concentrated in IPL sublaminae in which cells expressing the cognate gene form synapses. Predominant IPL sublamina marked by each IgSF member is drawn; sites of lower expression are shown with asterisks. ONL, outer nuclear layer; INL, inner nuclear layer; IPL, inner plexiform layer; GCL, ganglion cell layer; S1-S5, subdivisions of the IPL. '?' indicates that many retinal neurons are both Sidekick- and Dscam-negative, and may express other IgSF genes. Scale bars: b, $50 \mu \mathrm{m} ; \mathbf{c}, 25 \mu \mathrm{m}$; and d, $20 \mu \mathrm{m}$. identify appropriate cells following depletion. We tested previously described markers of RGC subsets ${ }^{10,23,24}$, and found that most Dscam-, Sidekick-1- and Sidekick-2-positive RGCs also expressed R-cadherin, cadherin-7 and calbindin, respectively (Supplementary Fig. 8 and data not shown). Thus, we could use these markers to assess lamina-specific connectivity in the absence of Dscam or Sidekicks.

Depletion of Dscam disrupted the laminar patterning of Rcadherin-positive processes in S5. Likewise, depletion of Sidekick-1 and Sidekick-2 disrupted laminar patterning of cadherin-7- and calbindin-positive processes, respectively, in S4. In all three cases, processes appeared defasciculated and extended beyond the boundaries of the sublamina in which they were normally confined (Fig. 3c). Several observations indicated that these effects were specific (Fig. 3c, Supplementary Fig. 7 and data not shown). First, no disruptions were seen in uninfected areas of the same retinae. Second, depletion of each IgSF member affected only the RGC subset marked by the coexpressed gene. Third, we saw no displacement of processes formed by choline acetyltransferase- and substance P-positive cells, which do not express Dscam or either Sidekick, although a small number of choline acetyltransferase-positive somata were sometimes displaced into the IPL following Dscam depletion (Supplementary Fig. 9). Fourth, R-cadherin and cadherin-7 were present not only in Dscam- and Sidekick-1-positive processes in S4 and S5, but also in Dscam- and Sidekick-1-negative processes in S2; only the former were affected by the interfering RNAs (see Supplementary Fig. 7 for examples and quantification). Finally, similar results were obtained with each of two interfering RNAs that depleted Dscam (Supplementary Fig. 6). Thus, Dscam, Sidekick-1 and Sidekick-2 are necessary for lamina-specific arborization of neurites in the IPL (Fig. 3d).

Next, we asked whether expression of Dscams or Sidekicks was sufficient to specify the laminar position of neurites in the IPL. Retroviral vectors such as those used to deliver interfering RNAs
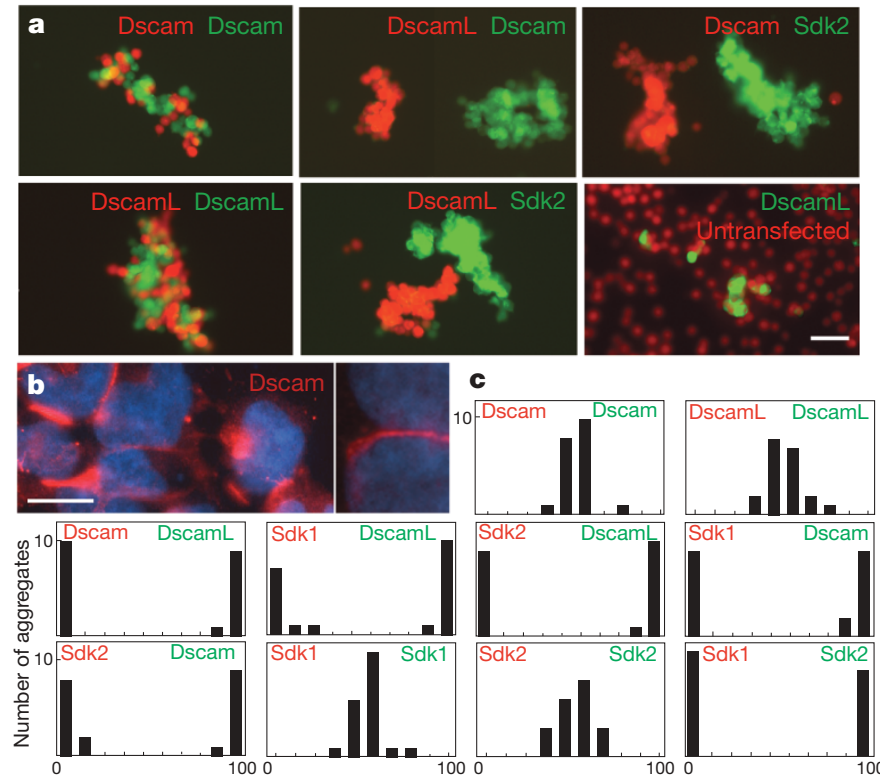

Percentage of green cells in aggregate

Figure 2 | Dscams and Sidekicks mediate homophilic adhesion but do not adhere to each other. Cells expressing each IgSF member were labelled red or green and incubated for $1 \mathrm{~h}$. The extent to which cells of the two colours mixed within aggregates was measured. a, Examples of homophilic but not heterophilic adhesion. Untransfected cells did not aggregate under these conditions. b, Dscam concentrates at sites of cell-cell apposition; low power figure at left, high power (twice magnified) at right. Nuclei marked with DAPI (blue).c, Percentage of green cells in each of 20 aggregates from each of 10 redgreen combinations. Scale bars: a, $50 \mu \mathrm{m} ; \mathrm{b}, 10 \mu \mathrm{m}$ (left) and $5 \mu \mathrm{m}$ (right). 
a

\begin{tabular}{|l|lll|l|}
\hline LTR & Viral genes & GFP & miR miR & LTR \\
\hline
\end{tabular}

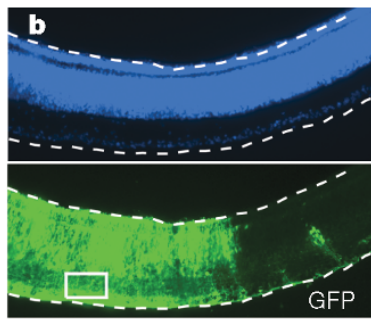

d
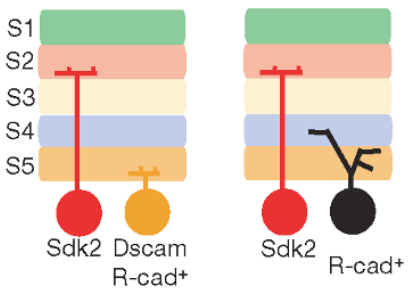

C Control
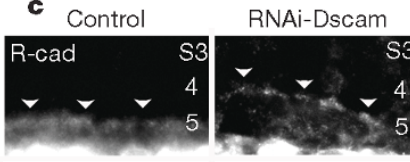

RNAi-Sdk2
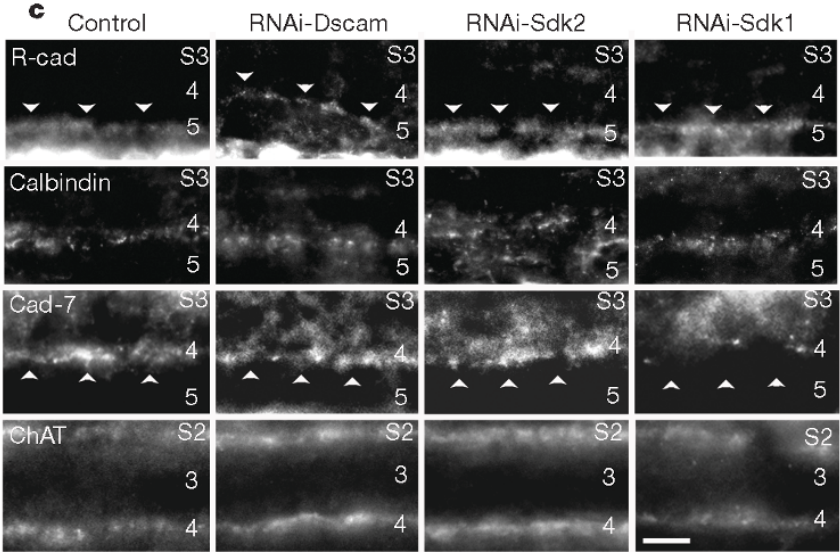

a

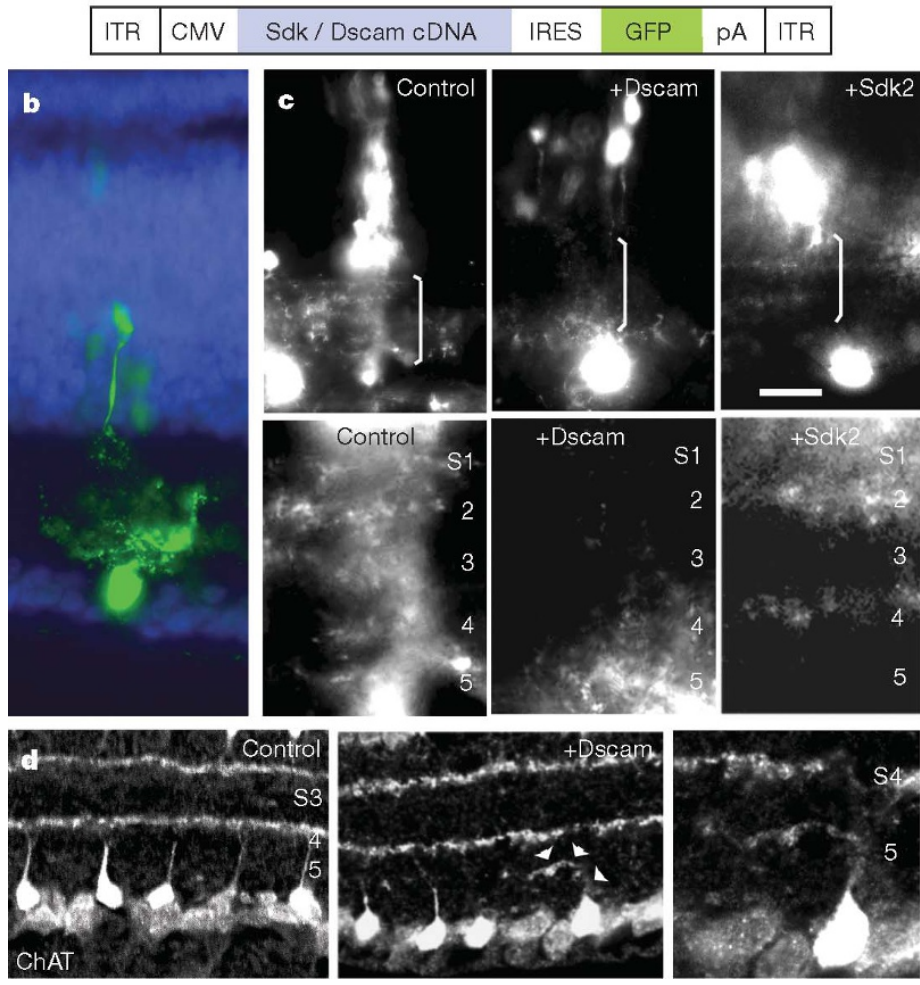

Figure 3 | Depletion of Dscam or Sidekick perturbs lamina-specific arborization. a, Retroviral vector. LTR, long terminal repeat; GFP, green fluorescent protein; $\mathrm{miR}$, artificial micro-RNAs directed at Dscam or Sidekick. b, Section from an E17 retina following retroviral introduction at E2. Large patches of retinal cells are infected, as shown by GFP expression (green). Blue, DAPI. Rectangle indicates approximate size and location of areas shown in c. c, IPL in areas infected by virus carrying control, Dscam, Sidekick-1 or Sidekick-2 interfering RNA, then immunostained as indicated with markers selectively coexpressed by RGCs that express Dscam (Rcadherin), Sidekick-1 (cadherin-7) and Sidekick-2 (calbindin). Depletion of Dscam, Sidekick-1 and Sidekick-2 leads to selective mislocalization of R-cadpositive processes in S5, and cadherin-7- and calbindin-positive processes in S4, respectively. Quantification of effects is detailed in Supplementary Fig. 7 legend. d, Summary of RNAi results, illustrated for Dscam. Scale bar in c indicates $50 \mu \mathrm{m}$ for $\mathbf{b}$ and $3 \mu \mathrm{m}$ for $\mathbf{c}$.

could not be used for this purpose because the size of the required cassette $(8-9 \mathrm{~kb})$ exceeds the packaging capacity of available vectors $^{22}$. Electroporation of plasmids ${ }^{10}$ was also problematic because expression levels declined greatly during the 2 -week interval between gene transfer (on E2) and scoring (on E17). We therefore used an alternative method of gene transfer utilizing PiggyBac transposon and transposase ${ }^{25,26}$. We adapted this insect-derived system to chicks, generated transposons encoding Dscams or Sidekicks plus GFP (Fig. 4a), introduced them into retina, and scored the laminar distribution of GFP-positive processes emanating from groups of GFPexpressing cells. When only GFP was introduced, processes were evenly distributed among IPL sublaminae, but introduction of a

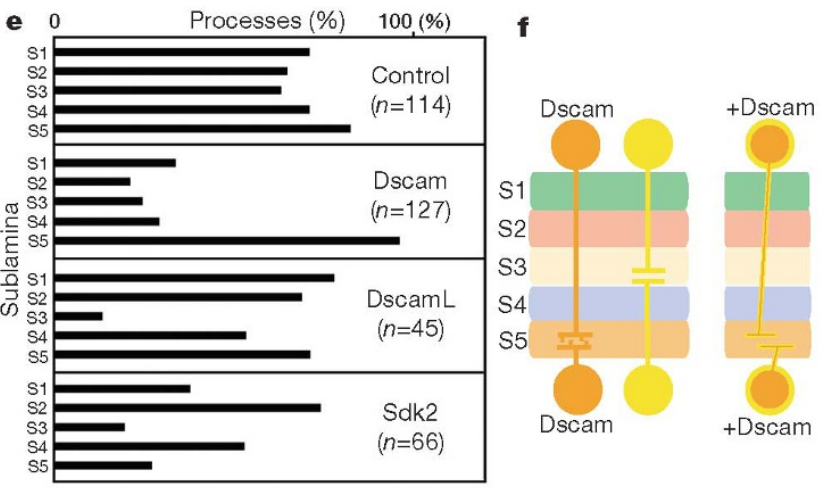

$\mathbf{g}$

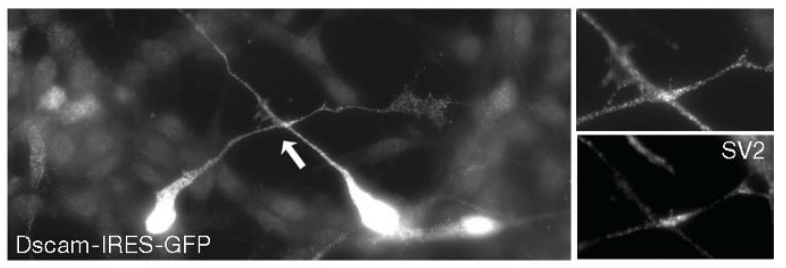

h

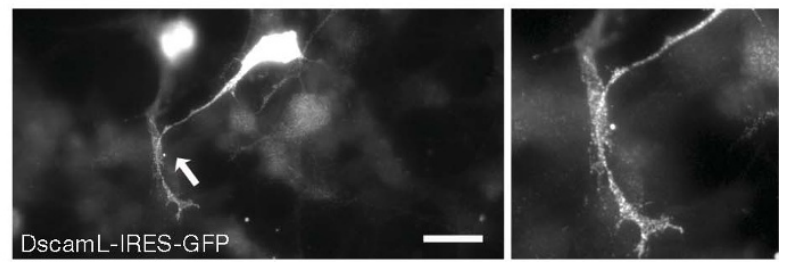

Figure 4 | Ectopic expression of Dscams or Sidekicks redirects laminaspecific arbors. a, PiggyBac transposon vector used to introduce Dscam or Sidekick plus GFP into retina. CMV, cytomegalovirus promoter and enhancer; ITR, internal terminal repeat; IRES, internal ribosomal re-entry site; $\mathrm{pA}$, polyadenylation signal. b, GFP-labelled cells in E17 retina following electroporation at E2. Blue, DAPI. c, GFP-labelled processes were distributed in all sublaminae when GFP alone was overexpressed. Ectopic expression of Dscam or Sidekick-2 shifted processes to Dscam-positive S5 or Sidekick-2positive S2, respectively. Brackets in top panels mark regions magnified in lower panels. d, Sections from retinae transfected with Dscam-GFP or GFP alone, stained for ChAT, which is expressed by a population of Dscamnegative amacrine cells. Dscam diverts ChAT-positive processes from S2 and
S4 to S5. Right panel shows region marked by arrowheads in centre panel. e, Laminar position of GFP-positive processes in electroporated retinae. Areas like those shown in $\mathrm{c}(n=45-127$ per construct) were analysed. $f$, Summary of gain-of-function results, illustrated for Dscam. $\mathbf{g}, \mathbf{h}$, Cultured neuronal cells were transfected with plasmids encoding Dscam-IRES-GFP $(\mathbf{g})$, or DscamLIRES-GFP (h) then stained with antibodies to GFP and the synaptic vesicle protein SV2. Regions indicated by arrows are shown at higher magnification at right. Neurites from transfected cells contacted each other, and sometimes accumulated SV2 at such points of contact or fasciculated with each other. Scale bar in c indicates $25 \mu \mathrm{m}$ for $\mathbf{b}, 50 \mu \mathrm{m}$ for c (top panels), $10 \mu \mathrm{m}$ for left and centre panels in d, and $3.3 \mu \mathrm{m}$ for right panel in d. Bar in $\mathbf{h}$ indicates $20 \mu \mathrm{m}$ for left panels in $\mathbf{g}$ and $\mathbf{h}$, and $10 \mu \mathrm{m}$ for right panels in $\mathbf{g}$ and $\mathbf{h}$. 
Dscam or Sidekick diverted processes to the sublamina in which the corresponding endogenous adhesion molecule is normally concentrated (Fig. 4b-e and data not shown). Cells ectopically expressing Dscam, for example, predominantly arborized in S5 (Fig. 4e). At least some of these processes had been destined for Dscam-negative laminae, as evidenced by their expression of choline acetyltransferase, which is normally confined to S2 and S4 (Fig. 4d). Likewise, cells that ectopically expressed DscamL avoided S3, which is normally DscamL-negative, and cells that ectopically expressed Sidekick-1 or Sidekick-2 sent processes predominantly to S2 and S4 (Fig. 4e, ref. 10 and data not shown). In all cases, both RGCs and interneurons were affected. Thus Dscams and Sidekicks are sufficient to strongly bias the laminar choices of retinal neurons (Fig. 4f).

These results suggest that Dscams and Sidekicks act as attractive or adhesive cues in the IPL, whereas Drosophila Dscams act as repulsive factors ${ }^{14,17-19}$. To explore this difference, we expressed Dscams or Sidekicks, along with GFP, in cultured neural cells. The transfected cells extended neurites that contacted each other, often accumulated synaptic vesicles at sites of contact, and sometimes fasciculated (Fig. 4g and h and Supplementary Fig. 10). The difference between the behaviour of vertebrate and Drosophila Dscams is likely to reflect context-dependence of signalling; many family of guidance molecules, including ephrins and netrins, act as attractants in some cells and repellents in others ${ }^{27,28}$.

In summary, we have shown that Dscams and Sidekicks mark and help to specify four parallel pathways through the inner retina. They mediate homophilic adhesion and are both necessary and sufficient to ensure sublamina-specific arborization in the IPL. We do not yet know the steps at which they act; they might regulate arborization of pre- and postsynaptic processes, target recognition, or stabilization of synaptic contacts. Although positioning of some amacrine cells is perturbed in Dscam mutant mice ${ }^{29}$, we do not believe that the defects we have seen are indirect consequences of such perturbations, in that RNA-interference-mediated loss of Dscam in chick does not lead to significant alterations in the distribution of amacrine cells that normally express this gene (data not shown). Moreover, positioning of amacrines is normal in early postnatal mouse Dscam mutants, at the time that synapses are forming in the IPL ${ }^{29}$. Although Dscam may play slightly different roles in the two species, we favour the idea that it has distinct effects on the two processes.

Dscams and Sidekicks mark only $\sim 60 \%$ of RGCs and a similar fraction of retinal interneurons (Supplementary Fig. 3 and Supplementary Table 1). Other adhesion molecules may exist that mark Dscam- and Sidekick-negative retinal neurons, subdivide groups of cells expressing each of these four IgSF members, and specify the tectal sublaminae in which axons of defined RGC subsets ${ }^{24}$ terminate. The retinotectal system may be useful for testing the idea that a complex network of recognition molecules mediates lamina-specific synaptic connectivity. Importantly, laminar specificity is a common feature of many circuits in the vertebrate brain and spinal cord ${ }^{30}$. As Dscams and Sidekicks are expressed by neuronal subsets in the brain (refs 6 and 9, and M.Y., unpublished results), similar networks may be used beyond the retina.

\section{METHODS SUMMARY}

Reagents. Monoclonal antibodies to chick Dscam and DscamL were generated using bacterially expressed recombinant proteins as immunogens. Full length cDNAs encoding Dscams and Sidekicks were cloned as described in Methods. For RNA interference, the DNA sequences specified in Methods were inserted along with GFP into the retroviral vector RCAS-BP (B). PiggyBac plasmids ${ }^{25}$ were obtained from M. J. Fraser (University of Notre Dame) and modified as described in Methods.

Histology. In situ hybridization and immunostaining were performed as described previously ${ }^{10,24}$.

Gene transfer in ovo. RCAS plasmids (for loss-of-function assays) or a 10:1:1 mixture of transposon, transposase and dsRed plasmids (for gain-of-function assays) were injected into the optic vesicles of stage 9-11 chick embryos.
Electroporation was with three square pulses of 7 V, $25 \mathrm{~ms}$. Embryos were fixed and sectioned at E17.

Cell culture. For adhesion assays, HEK293 cells (ATCC) were transfected with plasmids encoding Sidekick- or Dscam-GFP fusion proteins (EGFP-N1, Clontech). Stably expressing cells were selected, labelled with Cell tracker dyes (Invitrogen), and rotated at room temperature for $1 \mathrm{~h}$. For assays of neurite outgrowth, human neuroblastoma cells (SH-SY 5Y; ATCC) were plated on glass cover slips, transfected with Sidekick- or Dscam-IRES-GFP plasmid, then fixed and immunostained 3 days later.

Full Methods and any associated references are available in the online version of the paper at www.nature.com/nature.

\section{Received 31 August; accepted 12 November 2007.}

1. Ramón y Cajal, S. La rétine des vertébrés. La Cellule 9, 119-257 (1892); reprinted in The Vertebrate Retina (ed. Rodieck, R. W.) 775-904 (Freeman, San Francisco, 1974).

2. Masland, R. H. The fundamental plan of the retina. Nature Neurosci. 4, 877-886 (2001).

3. Wässle, H. Parallel processing in the mammalian retina. Nature Rev. Neurosci. 5, 747-757 (2004).

4. Roska, B. \& Werblin, F. Vertical interactions across ten parallel, stacked representations in the mammalian retina. Nature 410, 583-587 (2001).

5. Pang, J. J., Gao, F. \& Wu, S. M. Segregation and integration of visual channels: layer-by layer computation of ON-OFF signals by amacrine cell dendrites. J. Neurosci. 22, 4693-4701 (2002).

6. Yamakawa, K. et al. DSCAM: a novel member of the immunoglobulin superfamily maps in a Down syndrome region and is involved in the development of the nervous system. Hum. Mol. Genet. 7, 227-237 (1998).

7. Agarwala, K. L. et al. Down syndrome cell adhesion molecule DSCAM mediates homophilic intercellular adhesion. Brain Res. Mol. Brain Res. 79, 118-126 (2000).

8. Agarwala, K. L. et al. Cloning and functional characterization of DSCAML1, a novel DSCAM-like cell adhesion molecule that mediates homophilic intercellular adhesion. Biochem. Biophys. Res. Commun. 285, 760-772 (2001).

9. Yimlamai, D. et al. The zebrafish down syndrome cell adhesion molecule is involved in cell movement during embryogenesis. Dev. Biol. 279, 44-57 (2005).

10. Yamagata, M., Weiner, J. A. \& Sanes, J. R. Sidekicks: synaptic adhesion molecules that promote lamina-specific connectivity in the retina. Cell 110, 649-660 (2002).

11. Schmucker, D. et al. Drosophila Dscam is an axon guidance receptor exhibiting extraordinary molecular diversity. Cell 101, 671-684 (2000).

12. Wang, J., Zugates, C. T., Liang, I. H., Lee, C. H. \& Lee, T. Drosophila Dscam is required for divergent segregation of sister branches and suppresses ectopic bifurcation of axons. Neuron 33, 559-571 (2002).

13. Hummel, $T$. et al. Axonal targeting of olfactory receptor neurons in Drosophila is controlled by Dscam. Neuron 37, 221-231 (2003).

14. Zhan, X. L. et al. Analysis of Dscam diversity in regulating axon guidance in Drosophila mushroom bodies. Neuron 43, 673-686 (2004).

15. Zhu, H. et al. Dendritic patterning by Dscam and synaptic partner matching in the Drosophila antennal lobe. Nature Neurosci. 9, 349-355 (2006).

16. Chen, B. E. et al. The molecular diversity of Dscam is functionally required for neuronal wiring specificity in Drosophila. Cell 125, 607-620 (2006).

17. Zipursky, S. L., Wojtowicz, W. M. \& Hattori, D. Got diversity? Wiring the fly brain with Dscam. Trends Biochem. Sci. 31, 581-588 (2006).

18. Kidd, T. \& Condron, B. Avoiding the SCAMs. Neuron 54, 350-352 (2007).

19. Millard, S. S. et al. Dscam2 mediates axonal tiling in the Drosophila visual system. Nature 447, 720-724 (2007).

20. Zeng, Y., Cai, X. \& Cullen, B. R. Use of RNA polymerase II to transcribe artificial microRNAs. Methods Enzymol. 392, 371-380 (2005).

21. Chang, K., Elledge, S. J. \& Hannon, G. J. Lessons from Nature: microRNA-based shRNA libraries. Nature Methods 3, 707-714 (2006).

22. Hughes, S. H. The RCAS vector system. Folia Biol. (Praha) 50, 107-119 (2004).

23. Wohrn, J. C. et al. Cadherin expression in the retina and retinofugal pathways of the chicken embryo. J. Comp. Neurol. 396, 20-38 (1998).

24. Yamagata, M. et al. Labeled lines in the retinotectal system: markers for retinorecipient sublaminae and the retinal ganglion cell subsets that innervate them. Mol. Cell. Neurosci. 33, 296-310 (2006).

25. $\mathrm{Li}$, X. et al. The minimum internal and external sequence requirements for transposition of the eukaryotic transformation vector piggyBac. Mol. Genet. Genomics 266, 190-198 (2001).

26. Ding, S. et al. Efficient transposition of the piggyBac (PB) transposon in mammalian cells and mice. Cell 122, 473-483 (2005).

27. Poliakov, A., Cotrina, M. \& Wilkinson, D. G. Diverse roles of eph receptors and ephrins in the regulation of cell migration and tissue assembly. Dev. Cell 7, 465-480 (2004). 
28. Hong, K. et al. A ligand-gated association between cytoplasmic domains of UNC5 and DCC family receptors converts netrin-induced growth cone attraction to repulsion. Cell 97, 927-941 (1999)

29. Fuerst, P. G., Koizumi, A., Masland, R. H. \& Burgess, R. W. Neurite arborization and mosaic spacing in the mouse retina require DSCAM. Nature doi:10.1038/ nature06514 (this issue).

30. Sanes, J. R. \& Yamagata, M. Formation of lamina-specific synaptic connections. Curr. Opin. Neurobiol. 9, 79-87 (1999).

Supplementary Information is linked to the online version of the paper at www.nature.com/nature.
Acknowledgements We thank R. Burgess and colleagues for sharing data on mouse Dscam before submission, K. Hong for anti-sidekick-1 antiserum and M. J. Fraser (University of Notre Dame) for piggyBac plasmids. This work was supported by a grant from the NIH to J.R.S.

Author Contributions M.Y. and J.R.S. conceived the experiments. M.Y. performed the experiments. J.R.S. and M.Y. wrote the paper.

Author Information Reprints and permissions information is available at www.nature.com/reprints. Correspondence and requests for materials should be addressed to J.R.S. (sanesj@mcb.harvard.edu). 


\section{METHODS}

Sequence analysis. Homologies were calculated using EMBOSS 'water' pairwise alignment algorithms (http://www.ebi.ac.uk/emboss/align/index.html). Phylogenetic relationships of protein sequences were obtained from the Treefam database (http://www.treefam.org/). Multiple sequence alignment was performed with the ClustalW program (http://www.ebi.ac.uk/clustalw/ index.html). In all cases, alignments and calculations of similarity were done on the entire protein sequence.

Reagents. Mouse Dscam-like-1. A fragment of mouse Dscam-like-1 cDNA was amplified from the mKIAA1 132 plasmid (Kazusa DNA Research Institute) using the following primers: GGCCGCGGCCGCCCGCACGGCCGGCCACAGGACCACC and CGCGGTCGACAGGTCCCAGTGTGGAGCCCTTCTCC. This fragment was cloned into the NotI/SalI sites of pCMVscript (Stratagene). To complete its open reading frame, a BglII fragment of this plasmid was replaced by a cDNA amplified from mouse brain using the following primers: GGCCAGATCTCCGCACGGCCGGCCACAGGACCACC and CGCGAGATCTGTTGCCGTTGTGCTTGAGGGAGAC. The complete open reading frame from this construct was transferred to piggyBac transposon vector pXLBacIIRfA along with an IRES2-EGFP fragment (Clontech). To construct a Dscam-1GFP fusion protein, the sequence corresponding to extracellular plus transmembrane domains was amplified using primers CGCGCTCGAGCCGCACGGCCGGCCACAGGACCACC and GGCCGAATTCGCATTTCTGCCAAACTCTTTGCATC. The product was inserted between the EcoRI and XhoI sites of the EGFP-N1 vector.

Mouse Dscam. An AccI-NotI fragment from IMAGE clone 6410759 (ATCC) was cloned into the SalI/NotI sites of EGFP-N1. The AccI-AccI fragment was then replaced with cDNA amplified from mouse brain using primers GGCCGTCGACGTGGCTCGCTCGCTGGCTCGCTGGCT and CGACTCCAGCCGAGTTGTTGGCAGT. Transfer to the piggyBac transposon and construction of a GFP fusion construct were as above. The Dscam fragment for the fusion protein was generated using primers CGCGCTCGAGTGGCTCGCTCGCTGGCTCGCTGGC and GGCCGGGCCCGCATGAGCATTTCAGCTAAACTCTT.

Mouse Sidekick-1. A Sidekick fragment was amplified from an IMAGE clone and inserted into pCMVscript. The sequence GGCCGCGGCATGGCCCGCGCCCGGCCCTCGGT GGCGGGCGGCGGAGTCGCGGCGCCCCCTGAGC GAGCGGGGCCCGGGC was then inserted 5' of this fragment to complete the open reading frame. The sidekick-1-GFP fusion construct was made using primers CGCGCTCGAGAATTAACCCTCACTAAAGGGAAC and GGCCAAGCTTATCCAGGGTCACTGTCTCCTCCAT.

Mouse Sidekick-2. The NdeI-NotI fragment obtained from mKIAA1514 plasmid was cloned into the XhoI and NotI sites of EGFP-N1, and the XhoI-NdeI fragment was replaced by a cDNA amplified with primers GGCCCTCGAGTTATAAAGCAACTCGCAAAGAGG and CTGTTGTAGGCAGCCACCTCGATC. Transfer to the piggyBac transposon and construction of a GFP fusion construct were as above. The Sidekick-2-GFP fusion construct was made using primers CGCGCTCGAGCACTTTAGAGGACCCCTCGGTCA and GGCCGAATTCGACTGCTTTCATCCAAGCTTAGCAT.

Chicken Dscam. A fragment was amplified as above using primers ATCTTCAGCCTCCACCGTCTTTGT and TGCTTCTTGCTCGATCTGTGTCCT, and cloned into pGEM-Teasy vector. For bacterially expressed fusion proteins, extracellular and cytoplasmic domains were amplified using GCGAATTCGTGTCCTGGCTCCCTCCCCT plus CGCTCGAGAGATGTTCGCTGAGCAGTTG, and GGGCGAATTCACCGCATCCCCTCCCAAACCTCA plus GGCCCTCGAGGTAGGGGTTGTTTCCTTTCAAGTG. For ectopic expression, a cDNA amplified from E17 chicken retina total RNA using GCGCGGTCCGGAGATCTACACGCCAGTCTATATTTTGTCAATGCA and CGCGCTCGAGGCCATGCTCTTTTCGTTATACCAG was cloned into BspEI/Xhol site of APtag5 or FLAG-tagged APtag5 (GenHunter).

Chicken DscamL. For in situ hybridization, a $2 \mathrm{~kb}$ fragment was amplified from E17 chick retina using primers GAAACATGCGAAAGGCAGTGGGAA and GCTCGGAAGAGCTGATGAAGACAT. The fragment was cloned into pGEMTeasy vector (Promega), and used as a template for RNA polymerase. For bacterially expressed fusion protein, a fragment amplified using CCGAATTCGACATCGGCTACTACCTCTG and GCCTCGAGCGCACGGTTGAAGGCCTGAAC was cloned into pET23 vector (Novagen).

Chicken R-cadherin. The sequence was amplified from E17 chick retina using primers GGCCCGGCGGCCGCGGCACGGGGCGGCCGAGCGCAC and GGCCGGCTCGAGTAAGTGAGGTCAGTTCAATCCTCCT, and inserted into pCMVscript to generate riboprobes.

Histology. Histological methods are described in refs 10 and 24. Briefly, retinae were fixed in $4 \%$ paraformaldehyde in PBS, infiltrated with sucrose, frozen, and sectioned at $20 \mu \mathrm{m}$ in a cryostat. For immunohistochemistry, sections were stained successively with primary antibodies overnight and secondary antibody for 2-3 hours. For in situ hybridization, sections were hybridized with RNA probes that had been labelled by inclusion of digoxigenin- or fluorescein isothiocyanate-conjugated UTP in the RNA polymerase reaction. Following hybridization, probes were detected immunohistochemically using a tyramide signal amplification system (TSA-Plus, Perkin Elmer). Cells in the ganglion cell layer were identified as RGCs, based on their being Thy-1-positive and choline acetyltransferase-negative ${ }^{31,32}$.

Antibodies to R-cadherin (RCD2; ref. 33), cadherin-7 (CCD7) and SV2 were obtained from the Developmental Studies Hybridoma Bank. Other antibodies were: rabbit antibodies to choline acetyltransferase ${ }^{34}$, rat anti-substance $\mathrm{P}$ (Fitzgerald), rabbit anti-GFP (Chemicon), mouse anti-GFP (3E6, Invitrogen) and calbindin-28K (Swant). Rabbit polyclonal antibodies to mouse Sidekick-1 cytoplasmic domain were prepared by K. Hong in this laboratory. A monoclonal antibody to chick DscamL (DS1-96, IgG1) was generated by immunizing a $\mathrm{Balb} / \mathrm{c}$ mouse with a bacterially expressed extracellular domain of chick DscamL (see above). Antibodies to the extracellular domain of chick Dscam (DS2-44, IgG1, and rabbit polyclonal antibodies) and the cytoplasmic domain of chick Dscam (DS2-176, IgG1) were similarly generated by immunizing cognate fusion proteins (see above). Monoclonal antibody to Sidekick-2 (CS22, $\mathrm{IgG1}$ ) was described previously ${ }^{10}$. Fluorescence-conjugated secondary antibodies were from Invitrogen. For arbor analysis of GFP-filled retinal neurons, sections after fluorescence in situ hybridization using appropriate probes were further stained with chicken anti-GFP antibodies (Aves Lab, Inc.), and Alexa488 conjugated anti-chicken IgY secondary antibodies (Invitrogen). Arbors were analysed as described previously ${ }^{10,24}$.

Adhesion assay. Sidekick and Dscam extracellular and transmemebrane domains were cloned into pEGFPN1. HEK293 cells (ATCC) were transfected with these clones, and stably expressing cells were selected. Cells were labelled with appropriate Cell tracker (Invitrogen), dissociated with $0.05 \%$ trypsin in the presence of EDTA for $20 \mathrm{~min}$ at $37^{\circ} \mathrm{C}$. The reaction was stopped by egg white trypsin inhibitor. Cells were then divided into 24 well culture dishes in DulbeccoHamF12 medium supplemented with $1 \%$ BSA, $20 \mathrm{mM} \mathrm{HEPES}$ and $1 \mu \mathrm{g} \mathrm{ml}^{-1}$ DNase I, and rotated at room temperature for $1 \mathrm{~h}$.

Neuronal culture. Human neuroblastoma cells (SH-SY 5Y; ATCC: CRL-2266) were maintained in 1:1 Dulbecco-modified Eagle's minimal essential medium/ Ham F12 supplemented with $10 \%$ fetal calf serum. $5 \times 10^{5}$ cells were plated on a glass cover slip in a $35 \mathrm{~mm}$ dish, and transfected with plasmids in OptiMEM (Invitrogen) using the DMRIE-C transfection reagent (Invitrogen) for $15 \mathrm{~h}$. The medium was then replaced with fresh OptiMEM to promote neurite formation. Three days later, cultures were fixed with $4 \%(\mathrm{w} / \mathrm{v})$ paraformaldehyde in PBS, and immunostained with anti-GFP and anti-SV2.

RNA interference. The following DNA sequences were cloned into pcDNA6.2GW/EmGFP-miR (Invitrogen). Sdk-1-a, TGCTGAGCACTGGAAGATCCCTGCATGTTTTGGCCACTGACTGACATGCAGGGCTTCCAGTGCT; sdk-2-a, TGCTGTTGACAAAGGAGTGGGCTTTCGTTTTGGCCACTGACTGACGAAAGCCCTCCTTTGTCAA; dscam-b, TGCTGTACATGGACTTGCTGACGTCTGTTTTGGCCACTGACTGACAGACGTCAAAGTCCATGTA; dscam-c, TGCTGTTAACAGTGAGGTACATGGACGTTTTGGCCACTGACTGACGTCCATGTCTCACTGTTAA.

They were chained to generate two tandem copies as described in the manufacturer's protocol, and amplified along with EmGFP using primers CCGGCGGCATCGATACAAGTTTGTACAAAAAAGCAGGCT and GCGGCGGCATCGATACCACTTTGTACAAGAAAGCTGGGT. The fragments were cloned into pGEM-Teasy vector, then cloned into a unique ClaI site in the retroviral vector RCAS-BP (B). For injection, RCAS plasmids were concentrated to $5 \mu \mathrm{g}^{-1}$ in $10 \mathrm{mM}$ TrisHCl, pH 7.4, $1 \mathrm{mM}$ EDTA, mixed with Fast Green (final concentration $0.5 \%$ ), injected into optic vesicles of SPF chick embryos (Spafas), and then electroporated as described in Methods Summary.

PiggyBac transposon. PiggyBac plasmids ${ }^{25}$ were obtained from M. J. Fraser (University of Notre Dame). A transposase plasmid, pCMVpiggyBACorf, was generated from $\mathrm{p} 3 \mathrm{xP3}$-DsRed1-orf by removing DsRed sequences with XhoI and NotI. The transposon plasmid pXL-BacII-1 was modified by introduction of the acceptor segment RfA from GATEWAY (Gateway Conversion System, Invitrogen) between SmaI and EcoRV sites, generating pXL-BacIIRfA. Full length cDNAs and IRES2-EGFP sequence were cloned into pCMVscript (Stratagene) or pEGFP-N1, then cassettes containing CMV promoter, cDNA, IRES2-EGFP and SV40 polyadenylation signal were cloned into pXL-BacII-RfA using the following attB1/B2 primers: GGGGACAAGTTTGTACAAAAAAGCAGGCTTAGTTATTAATAGTAATCAA, GGGGACCACTTTGTACAAGAAAGCTGGGTTTAAGATACATTGATGAGTT. Plasmids were concentrated and electroporated as above.

31. Spira, A. W. et al. Localization of choline acetyltransferase-like immunoreactivity in the embryonic chick retina. J. Comp. Neurol. 260, 526-538 (1987). 
32. Barres, B. A. et al. Immunological, morphological, and electrophysiological variation among retinal ganglion cells purified by panning. Neuron 1, 791-803 (1988)

33. Inuzuka, H., Miyatani, S. \& Takeichi, M. R-cadherin: a novel $\mathrm{Ca}(2+)$-dependent cell-cell adhesion molecule expressed in the retina. Neuron 7, 69-79 (1991).

34. Johnson, C. D. \& Epstein, M. L. Monoclonal antibodies and polyvalent antiserum to chicken choline acetyltransferase. J. Neurochem. 46, 968-976 (1986). 